\title{
COMPORTAMENTO AGRESSIVO E BUSCA DE SENSAÇÃO EM JOVENS
}

\author{
Agressive Behavior and Sensation \\ of Seeking in Young
}

\author{
Nilton Soares Formiga ${ }^{1}$ \\ Carmem Plácido Cavalcante 2 \\ Thaís Teixeirade Vasconcelos Araújo ${ }^{2}$ \\ Silvana Lima ${ }^{2}$ \\ RobertaSantana ${ }^{2}$
}

\section{Resumo}

O problema da delinqüência juvenil tem interessado as ciências sociais e humanas. O construto da busca de sensações, que enfatiza as diferenças individuais, mostrando que a partir da perspectiva personalística a avaliação do grau com que os jovens pretendem viver experiências em correr riscos e sua predisposição às sensações de novidades e intensidades vem contribuir para explicação do problema. 504 jovens, entre 11 e 20 anos, responderam o questionário de comportamento agressivo e da busca de sensação. A busca da sensação de intensidade e novidades relacionou-se positivamente com o comportamento agressivo físico, verbal, raiva e hostilidade. Os traços de personalidade, no construto da busca de sensação, ainda têm contribuído na explicação da conduta juvenil, especialmente aquelas permeadoras da delinqüência. Tais resultados apontam na direção de uma orientação das atitudes conscientes frente à excitação e investimento de atividades permeadoras de risco.

Palavras-chave: Comportamento agressivo; Personalidade; Jovens.

\footnotetext{
Mestre em psicologia social. Atualmente, é doutorando na Universidade Federal da Paraíba e docente na Universidade Estadual da Paraíba. João Pessoa, PB. e-mail: nsformiga@yahoo.com

2 Alunas do curso de Psicologia na Universidade Federal da Paraíba. e-mails: carmemplacido@yahoo.com.br; thaisteixeira@hotmail.com; silvanalima@yahoo.com.br; robertasantana@hotmail.com
} 


\section{Summary}

The problem of the juvenile delinquency has been interest for the social and human science. The construct seeking of sensation has emphasized the individual differences, contributing starting from the perspective personalistic, the evaluation of the degree with that the youths intend to live experiences in running risks and its predisposition to sensations of novelties and intensities. 504 young, between 11 and 20 years, they answered the aggressive behavior and of sensation seeking. The seeking sensation the run of the risk and predisposition the novelty and intensity sensations, it correlated positively to the physical, verbal aggressive behavior, anger and hostility. The personality traits, in the sensation seeking construct, still comes to contribute in the explanation of the juvenile conduct, especially those related the deliquency. Such results point in direction of an orientation of the attitudes conscious front the excitement and investment of activities of the risk.

Keywords: Aggressive behavior; Personality; Young.

\section{Introdução}

Nos últimos anos, é possível observar o quanto jovens das classes média e alta têm manifestado condutas com características delinqüentes, as quais vêm se tornando salientes em nosso cotidiano urbano, principalmente quando acompanhamos as notícias na mídia em geral relatando, por exemplo, condutas que vão desde as formas de organização social desviante adotada por esses jovens (as chamadas gangues), aos jogos de diversão manipuladores de atos violentos e criminais, as balbúrdias em festas, vandalismo e ao consumo de drogas lícitas e ilícitas (Formiga \& Gouveia, 2005). Frente a esse fato, algumas explicações são apontadas - por exemplo, estrutura e funcionalidade da família, valores humanos e culturais, desenvolvimento moral, hábitos de lazer e estrutura e traços de personalidade - em relação ao quanto esse fenômeno poderá vir a ser tão freqüente na dinâmica juvenil caso tardem a compreensão e soluções urgentes (Coelho Junior, 2001; Formiga \& Gouveia, 2005; Frías-Armenta, López-Escobar \& Díaz-Méndez, 2003; Sobral, 1996).

Apesar das variáveis apontarem, cada uma a seu mérito, em direção das soluções quanto à predição de um problema social tão grave, ainda tem sido promissor o poder explicativo das teorias sobre a personalidade. Independente da linha teórica que venha defender, ideográfica ou nomotética ${ }^{3}$ ou quanto ao seu foco filosófico em relação ao inato ou adquirido no que se refere à organização desse construto no ser humano, eles têm trazido indicadores, empíricos e teóricos, em termos das idiossincrasias do sujeito e a influência na conduta social humana (Benet-Martínez, \& John, 1998; Gazzaniga, \& Heatherton, 2005). Recentemente, com a divulgação e investida dos pesquisadores em relação ao modelo dos cinco grandes fatores para explicação do comportamento humano, esse modelo dos traços vem obtendo, de acordo com Pervin e John (2004, p. 212), "evidências a partir de três áreas principais: análise fatorial de grandes conjuntos de termos lingüísticos relacionados com os traços, pesquisas interculturais testando a universalidade das dimensões dos traços e a relação entre questionários de traços e outros questionários e avaliações." (Cloninger 1999; Hutz, et al, 1998).

Com isso, a concepção da personalidade a partir dos traços vem tratar da conduta humana não como questões patológicas, mas em termos da díade genética/meio ambiente, a qual implicaria em características individuais consistentes do comportamento, exibido pelo indivíduo em diversas situações normalmente concebido como disposições (Costa \& McCrae, 1992; Saudino \& Plomin, 1996). Para Formiga, Yepes e Alves (2005), o que vem gerar taxonomias que permita ao sujeito expressar, por meio das condutas, formas específicas para si e os outros quando em interação social, situação em que na maioria das vezes atendese a desejabilidade social; isto é, o sujeito procura parecer melhor para os outros, descrevendo-se como gostaria que fosse descrito para quem o observa, justamente porque essa auto-imagem exigida se deve a uma co-dependência dos "papéis" sociais representados por cada um de nós

\footnotetext{
Diz respeito ao estudo do comportamento individual com a finalidade de elaborar leis de comportamento, nas quais todas as pessoas pudessem ser encaixadas, ou enfatizar ao estudo do indivíduo procurando conhecer suas singularidades.
} 
(Queiroga, Formiga, Jesus, Gouveia \& Andrade, 2001). Todavia, faz-se necessário esclarecer que não se pretende no presente estudo aprofundar uma discussão epistemológica quanto ao estudo dos traços de personalidade, afinal, é possível encontrar muitos manuais de psicologia (por exemplo, Allport, 1973; Díaz, 1998; Pervin \& John, 2004; Robinson, Shaver \& Wrightsman, 1991) que abordam detalhadamente o problema teórico e metodológico, afinal esse não é o objetivo principal do estudo.

Essa linha de pesquisa sobre os traços de personalidade não é nova na Psicologia, mas, nos últimos anos, os construtos estruturais ou dos traços vem sendo retomados e acrescentando informações na compreensão preditiva do comportamento humano, principalmente, em relação àqueles que permeiam a violência (por exemplo, anti-sociais e delitivas, comportamento agressivo, uso de drogas, etc.) (Paunonen, 1998; Sobral, 1996; Stephenson, 1990). Apesar de existirem inúmeras variáveis que possam compor o fenômeno da delinqüência na literatura em geral, o objetivo principal desse estudo tratará do comportamento agressivo, que na concepção de Buss e Perry (1999) estrutura-se como agressão física, agressão verbal, raiva, hostilidade e sua explicação a partir dos traços de personalidade, enfatizando o construto busca de sensação; para uma compreensão didática dessas variáveis, logo abaixo, viu-se a necessidade de esclarecer as variáveis a serem estudadas no presente trabalho, as quais já foram desenvolvidas e avaliadas por outros autores (ver Formiga e Gouveia, 2003; Frías-Armenta, López-Escobar, \& Díaz-Méndez, 2003; Zhang, Welt, \& Wieczorek, 1999).

\section{O comportamento agressivo}

Nas ciências humanas, sociais e biológicas, o estudo da agressão é apresentado sob uma diversidade de termos na literatura, podendo ir da sua sobreposição as distinções frente ao que é esse fenômeno (Kristensen, Lima, Ferlin, Flores, \& Hackmann, 2003). Apesar das diversas variáveis poderem apontar em direção de explicações quanto à origem e motivo da conduta agressiva no ser humano - as quais podem ir desde as mudanças hormonais, fatores inconscientes, aprendizagem social, modelos ou esquemas mentais, etc. - a maioria de seus determinantes são apresentados a partir de vetores sociais, pois, independente de assumirem uma identidade com a agressão, eles somente ocorreram na presença, concreta ou simbólica, de outras pessoas, isto é, nas relações psicossociais. De modo geral a agressão pode ser definida como qualquer comportamento que tem o objetivo de prejudicar ou ferir física ou psicologicamente algum outro ser humano, podendo ser expresso de forma instrumental ou expressiva e que a pessoa-alvo desse comportamento deseja evitar (Aronson, Wilson, \& Akert, 2002; Michener, Delamater, \& Myers, 2005).

Muitas hipóteses foram elaboradas a fim de avaliar motivo, causa e origem que levam as pessoas a serem agressivas. ${ }^{4}$ Anderson e Buschmam (2002), Buss e Perry (1999) e Kristensen, et al. (2003) fazem uma ótima revisão sobre a explicação desse fenômeno, por exemplo: a teoria da frustração-agressão, elaborada por Miller, Sears, Mowrer, Doob e Dollard (1941, citado em Kristensen, et al., 2003) visava explicar etiologicamente a agressão, estabelecendo que quando o indivíduo observava a agressão ele deveria suspeitar das possibilidades com que o organismo ou grupo é confrontado com frustração e que ao sofrer interferência com hábitos individuais ou grupais, haveria possibilidade de manifestar a agressão; outra teoria, a aprendizagem social desenvolvida por Albert Bandura, propunha que a agressão é aprendida e perpetuada pelo sistema de reforçamento positivo ao longo da vida; uma outra, a teoria da cognição social, enfatiza a importância de percepção e interpretação dos estímulos do meio ambiente que determinam se o indivíduo responderá ou não agressivamente a um estímulo.

Afinal, muitas são as pesquisas que levantam hipóteses a respeito das variáveis explicativas da agressão; independente da perspectiva teórica que possa ser enfatizada, atualmente, vem sendo divulgada na comunidade científica a proposta de Buss e Perry (1999) em termos do comportamento agressivo, a qual será utilizada no presente estudo. Originado do inventário de Hostilidade de Buss-Durkee, o questionário de agressão tem como objetivo avaliar o

\footnotetext{
Não pretendemos nos deter concisamente nestas teorias, pois, diversos manuais, tanto de introdução a psicologia quanto de psicologia social, psicobiologia e neuropsicologia, entre outros, tratam detalhadamente dessas teorias. A intenção deste parágrafo trata-se apenas de abordar, informativamente, os primeiros estudos sobre o fenômeno da agressão.
} 
grau de variação na pontuação do comportamento juvenil agressivo (Andreu, Pena, \& Graña, 2002); fato esse que, segundo Buss e Perry (1999), é dimensionado em agressão física, agressão verbal, raiva, hostilidade, dimensões estas que podem caracterizar a forma instrumental e expressiva desse construto.

De fácil aplicação e baixo custo, esse instrumento tem conseguido uma validade internacional quanto à consistência e fidedignidade das dimensões do comportamento agressivo proposto pelos autores (Andreu, Pena, \& Graña, 2002; Collani, \& Werner, 2005; Fossati, Maffei, Acquarini, \& Di Ceglie, 2003). Além de segurança estatística, nesses referidos estudos, vem se encontrando uma relação interna entre os fatores do comportamento agressivo, permitindo pensar uma interdependência entre as dimensões do construto; provavelmente, a maior pontuação em uma das dimensões do comportamento agressivo repercutirá no aumento nas outras dimensões. Sendo assim, esse fenômeno poderia ser pensando em termos de um sistema auto-influenciador, rompendo com a noção de agressão como construto linear.

No Brasil, Formiga (2005) e Formiga, Trigueiro, Melo e Dourado (2006) desenvolveram um estudo com esse instrumento. Apesar de ser algo preliminar e exploratório numa amostra de jovens não delinqüentes, foram identificadas as quatro dimensões destacadas pelos autores citados no parágrafo acima. Atualmente, este estudo está sendo ampliado, a partir de uma análise semântica e metodológica em uma população brasileira maior no nordeste, sul e sudeste do país. Sendo assim, tendo o comportamento agressivo, teórica e empiricamente, adquirido garantia mensurável ainda se discute a origem e motivos que levam as pessoas a variarem seu comportamento agressivo (Andreu, Pena, \& Graña, 2002; Bryant, \& Smith, 2001; Buss, \& Perry, 1999; Collani, \& Werner, 2005). Para isso, é hipotetizado que o construto da agressão possa ser influenciado pelos traços de personalidade, especificamente, pelo construto busca de sensação.

\section{A abordagem dos traços $e$ as condutas de risco}

$\mathrm{Na}$ psicologia, a personalidade tem sido explorada como um construto capaz de explicar as diferenças individuais, proporcionando um marco teórico nos estudos a respeito das idiossincrasias e estabilidade da conduta de cada pessoa (Ávila,
Rodríguez, \& Herrero, 1997; Barbaranelli, \& Caprara, 1996). Esse fato possibilita, a partir das características individuais, uma avaliação científica ou de senso comum em situações variadas quando se pretende predizer reações ou disposições futuras das pessoas (Gazzaniga, \& Heatherton, 2005; Paunonen, 1998; Peabody, 1987; Trzop, 2000). Desta forma, o conhecimento da personalidade não somente pode contribuir na organização das relações interpessoais, como também apontar em direção dos fatores de proteção frente a essas relações (McAdams, 1992).

$\mathrm{Na}$ literatura científica é possível encontrar uma diversidade de estudos que enfatizam a relação entre personalidade e conduta humana, principalmente, condutas que visam à quebra de normas sociais. Por exemplo, segundo Eysenck (1981), o jovem que apresenta uma conduta antisocial em geral possui traços de neuroticismo e impulsividade; resultado semelhante foi achado por Tranah, Harnett e Yule (1998), quando trataram do distúrbio de condutas, verificando correlação positiva e significativa com os traços de psicoticismo e neuroticismo. Apesar dos resultados refletirem em termos dos aspectos desestruturantes da personalidade, mesmo com base nos estudos dos traços, eles revelam um sujeito não sociável, emocionalmente negativo, possuidor de baixa autoestima, entre outras características não adaptativas e de fraca habilidade social. Da mesma maneira, Romero, Luengo e Sobral (2001) observaram em jovens espanhóis sem história clínica de delinqüência, que as dimensões da personalidade (por exemplo, psicotismo, impulsividade, busca de sensação e extroversão) estão fortemente correlacionadas com a quebra de normas, indicando assim que elas constituem um fator de risco para o comportamento anti-social.

Seguindo semelhante raciocíonio, em um estudo realizado por Heaven, Newbury e Wilson (2004), os quais consideraram a dimensão da escala de psicotismo de Eysenck em jovens não delinqüentes de 11 a 16 anos de idade, foi observado que este traço predisse uma diversidade de comportamentos delinqüentes (por exemplo, uso de droga, dano de propriedade, entre outros, etc.). Formiga, Teixeira, Curado, Lüdke e Oliveira (2003) e Formiga et al. (2005) chegaram a resultado próximo a esse, a partir da teoria dos cinco grandes fatores da personalidade, observando que as dimensões personalísticas de extroversão 
(relacionados à atividade e energia, dominância e emoções positivas) e abertura à mudança (comportamentos aberto a novas experiências, experimentar coisas novas) explicaram, positivamente, as condutas anti-sociais e delitivas, já a dimensão de agradabilidade (orientação prósocial com os demais, altruísmo, confiança) explicou, negativamente, as condutas desviantes.

Mesmo que esses estudos contribuam em direção da explicação da conduta de risco (por exemplo, conduta anti-social, delitiva, uso de drogas, etc.) apresentada pelos jovens como uma tendência ou predisposição ao delinqüir a partir de alguns traços da personalidade, avaliada por meio de instrumento como o 16-PF, NEO, PEN, OCEAN ou cinco grandes fatores (Big Five) reflete-se em termos das polarizações entre traços de impulsividade e experimentação e traços de respeito humano e social e consciência dos limites, sendo eles, respectivamente, capazes de explicar os fatores de risco e proteção (Anastasi \& Rubina, 2000; Cordero, 1997; Engler, 1984). Desta forma, o objetivo do presente estudo visa uma explicação em relação à predisposição do jovem em viver intensamente as experiências, entendidas como busca de sensação, e sua relação com o agir agressivo.

O foco nessa perspectiva é enfatizado porque na literatura em geral e nossas relações cotidianas considera-se que o adolescente tem uma necessidade latente em expandir seu mundo ideal e "real" por meio do comportamento de reivindicação e instrumentalidade, estando disposto a convites pessoais ou sociais para viver novas descobertas e experimentá-las intensamente, concebendo, com isso, que esses conglomerados comportamentais possam ser caracterizados como a busca de sensação (Arnett, 1994; Omar, \& Uribe, 1998; Michel, et al, 1999; Zuckerman, 1971).

Considerado um traço personalístico, a busca de sensação teve seu estudo iniciado por Zuckerman (1971), o qual definia o construto como uma necessidade de viver experiências complexas e de novidades, apenas pelo desejo de afrontar riscos físicos e sociais, com o intuito de satisfazer suas necessidades pessoais (Zuckerman, Eysenck, \& Eysenck, 1978). Desta forma, o construto busca de sensações não apenas é tido como necessidade individual de experimentar situações de risco em termos da proposta por M. Zuckerman (1971), mas, tendo estes traços sua inserção na socialização, a qual vem sendo avaliada com bastante ênfase nos últimos anos. Essa dimensão personalística, segundo Arnett (1994; 1996), pretende compreender o comportamento juvenil, principalmente, aqueles que caracterizam transgressões de normas sociais, como variações do comportamento de risco a partir da investida do jovem na busca de novas experiências e emoções intensas (Mussen, Conger, Kagan, \& Huston, 1995).

De acordo com Arnett (1994), partindo da perspectiva de M. Zuckerman, faz-se referência aos limites tanto na concepção do construto quanto de sua instrumentalização e seleção dos itens, propondo um modelo alternativo, no qual defende que a busca de sensação varia entre intensidade e novidade e não apenas em termos da complexidade condutual como concebia Zuckerman, mas que a dimensão do traço de personalidade deveria ser enfatizada sobre o processo de socialização, o qual tornaria capaz de modificar predisposições biológicas, respondendo ao dilema genéticoambiente. Um outro aspecto apontado por Arnett é que a busca de sensação se desenvolve a partir do interesse de experiências novas e intensas e não necessariamente apenas em correr risco pelo risco (Omar, \& Uribe, 1998). Ao considerar essas variáveis, é possível apresentar mais uma peça no quebra-cabeça do problema da delinqüência, principalmente, em relação aos construtos que avaliam traços de personalidade os quais têm oferecido bastante resposta entre elas.

No Brasil, poucos estudos têm relacionado a busca de sensação e comportamento agressivo; a grande parte dos estudos em outros países enfatiza instrumentos como o NEO-PI-R, Big Five, entre outros menos conhecidos; dos estudos encontrados sobre essas relações, estes não utilizam o proposto por Arnett (1994). Resumidamente, os estudos de Caprara, Barbaranelli e Zimbardo (2003), Sharpe e Desai (2001), Tremblay e Ewart (2005), recentemente, Collani e Werner (2005), observaram que o traço de personalidade neuroticismo - aqueles que, segundo Benet-Martínez e John (1998), dizem respeito à estabilidade emocional com afetos negativos, incluindo ansiedade, tristeza, irritabilidade e tensão nervosa - relaciona-se com o comportamento agressivo físico, a hostilidade e a raiva. Apesar de não ter encontrados estudos que abordassem as variáveis em questão, algo merece destaque: ainda é possível observar a contribuição dos construtos dos traços de personalidade, em suas variadas teorias, na explicação das condutas 
permeadoras da delinqüência. Porém, frente ao novo construto abordado, pretende-se apontar em direção não somente da perspectiva individual, mas também do processo de socialização na qual a personalidade é formada; para tanto, o objetivo do presente estudo é avaliar a relação entre busca de sensação e comportamento agressivo em jovens.

\section{Método}

\section{Amostra}

504 jovens compuseram a amostra, os quais estiveram distribuídos igualmente no nível escolar fundamental e médio da rede privada e pública de educação na cidade de Palmas - TO, com idades entre 11 e 20 anos e uma renda econômica média de 820,00 reais. Os respondentes foram de ambos os sexos, predominando ligeiramente a participação de mulheres (53\%). Tal amostra foi não probabilística e sim intencional, pois além do propósito de garantir a validade externa de alguns instrumentos da pesquisa, era assegurada a possibilidade de realizar as análises estatísticas que permitissem estabelecer as relações entre as variáveis hipotetizadas no estudo.

\section{Instrumento}

Os participantes responderam um questionário composto das seguintes medidas:

Questionário do comportamento agressivo - Este instrumento, composto por 26 itens, distribuídos em quatro fatores: agressão física (por exemplo, "Se alguém me bater, eu bato de volta"), agressão verbal (por exemplo, "eu não consigo ficar calado(a) quando as pessoas discordam de mim") raiva (por exemplo, "algumas vezes me sinto como uma bomba preste a explodir") e hostilidade (por exemplo, "eu desconfio de pessoas estranhas que são amigáveis demais") foi proposto por Buss e Perry (1999), o mesmo foi validado em uma população de infratores por Williams, Boyd, Cascardi e Poythress (1996), encontrando uma estrutura composta por 26 itens distribuídos como agressão física, agressão verbal, raiva, hostilidade. Formiga, Trigueiro, Melo e Dourado (2006) e Formiga et al. (2005), no Brasil, aplicaram essa escala como estudo exploratório numa amostra de jovens não delinqüentes, identificando, também, as quatro dimensões destacadas por Buss e Perry (1999). Assim, utilizou-se a adaptação dessa versão no presente estudo, na qual os itens eram respondidos numa escala de resposta do tipo Likert com os extremos variando de $\mathbf{1}=$ Discordo Totalmente a $\mathbf{5}=$ Concordo Totalmente.

Inventário de Busca de sensação. Este instrumento, construído por Arnett (1994) e Omar e Uribe, (1998) trata-se de uma escala composta por vinte itens, os quais compõem duas subescalas: a busca intensidade (por exemplo, "quando escuto música, eu gosto de escutá-la bem alto"; "gosto de assistir filmes onde tem muita explosão e batidas"; "deve ser muito excitante estar brigando numa guerra") e a busca da novidade (por exemplo, "seria interessante casar-me com alguém de um país estrangeiro"; "pensou que é divertido falar, atuar ou se mostrar mesmo da frente das pessoas"; "gostaria muito de viajar para lugares longe e desconhecidos") na estimulação dos sentidos, cada uma com dez itens. Para respondê-la, a pessoa utilizava uma escala de resposta tipo Likert com quatro pontos $(\mathbf{1}=$ não me descreve em nada; $\mathbf{2}=$ descreve-me em alguma medida; $\mathbf{3}=$ descreve-me bem e $\mathbf{4}=$ descreve-me totalmente), devendo indicar nesta o quanto cada um dos itens descreve sua conduta habitual.

Caracterização Sociodemográfica. Foram elaboradas perguntas que contribuíram para caracterizar os participantes deste estudo (por exemplo, sexo, idade, classe social), bem como realizar um controle estatístico de algum atributo que possa interferir diretamente nos seus resultados.

\section{Procedimento}

Procurou-se definir um mesmo procedimento padrão que consistia em aplicar os instrumentos coletivamente em sala de aula, em escolas de ambas as áreas urbanas da cidade de Palmas-TO. Colaboradores com experiência metodológica e ética ficaram responsáveis pela coleta dos dados; após conseguir a autorização tanto da diretoria da escola quanto de professores responsáveis pela disciplina, os aplicadores se apresentavam na sala de aula como interessados em conhecerem as opiniões e os comportamentos das pessoas no dia-a-dia, solicitando a colaboração voluntária dos estudantes no sentido de responderem um questionário breve. Para isso, foilhes dito que não havia resposta certa ou errada e que respon-dessem individualmente, bem como quanto a 
sua não obrigação para essa tarefa, sendo que ao pegar o instrumento e ao iniciar sua leitura e resposta, se quisesse devolvê-lo não teria nenhum problema.

Mesmo com esse esclarecimento, a todos foi assegurado o anonimato das suas respostas, comunicando-lhes que elas seriam tratadas em seu conjunto; apesar do questionário ser auto-aplicável, contando com as instruções necessárias para que possam ser respondidos, os colaboradores estiveram presentes durante toda a aplicação para retirar eventuais dúvidas ou realizar esclarecimentos que se fizessem indispensáveis, não interferindo na lógica e compreensão da resposta dos respondentes. Um tempo médio de 30 minutos foi suficiente para concluir essa atividade. Quanto à análise dos dados, utilizou-se o pacote estatístico SPSSWIN, em sua versão 11.0, para tabulação e realização das análises estatísticas descritivas e os coeficientes de correlação $r$ de Pearson.

\section{Resultados e discussão}

Antes de atender o objetivo principal do presente estudo, resolveu-se avaliar a relação interna entre os construtos; inicialmente, o comportamento agressivo e em seguida a busca de sensação. A partir de uma correlação de Pearson (r), observou-se uma relação direta e significativa entre o comportamento agressivo físico com a agressão verbal, raiva e hostilidade. A agressão verbal também se relacionou com a raiva e hostilidade, e esta com a raiva (ver Tabela 1 ). Assim, além desses resultados corroborarem outros estudos que utilizaram o mesmo instrumento, os quais encontraram correlações semelhantes na mesma direção entre os fatores do comportamento agressivo, esses resultados permitem refletir a existência de um sistema correlacional entre os comportamentos agressivos (Collani, \& Werner, 2005; Buss, \& Perry, 1999; Fossati, et al, 2003; Meesters, Muris, Bosma, Schouten, \& Beuving, 1996). Referindose aos escores correlacionais encontrados entre essas dimensões é bem possível que o indivíduo que apresente um desses comportamentos agressivos, provavelmente, apresentará um ou mais de um dos outros comportamentos.

Parece que esse construto está para além de uma perspectiva linear; isto porque, em termos da agressão, esta poderia ser interpretada não somente

\section{Tabela 1 - Correlação interna dos comportamentos agressivos}

\begin{tabular}{lcccc}
\hline $\begin{array}{l}\text { Comportamentos } \\
\text { Agressivos }\end{array}$ & $\mathbf{1}$ & $\mathbf{2}$ & $\mathbf{3}$ & 4 \\
\hline Físico & $-\ldots$ & & & \\
Verbal & $0,19^{*}$ & $-\ldots$ & & \\
Raiva & $0,54^{*}$ & $0,33^{*}$ & $-\ldots$ & \\
Hostilidade & $0,26^{*}$ & $0,55^{*}$ & $0,40^{*}$ & $-\ldots$ \\
\hline
\end{tabular}

Nota: ${ }^{*} \mathrm{p}<0,01$.

como uma dimensão global, mas sim como um potencial continuum entre suas dimensões, influenciadose a partir de seu grau de intensidade comportamental em cada uma dessas dimensões. Quanto ao construto busca de sensação, estes também se mostraram interrelacionados, a saber: a busca de sensação de novidade $e$ intensidade $(\mathrm{r}=0,42 ; \mathrm{p}<0,001)$ relacionaram-se tanto entre si quanto com o somatório total desse construto, etiquetado como busca de sensação (BS) (respectivamente, $\mathrm{r}=0,40, \mathrm{r}=0,38 ; \mathrm{p}<0,001$ ).

A partir desses resultados, passou-se a contemplar o objetivo principal do presente estudo - avaliar a relação entre comportamento agressivo e busca de sensação - assim, a busca de sensação intensa relacionou-se, positivamente, ao comportamento agressivo físico $(\mathrm{r}=0,41, \mathrm{p}<0,001)$, agressão verbal $(\mathrm{r}=$ $0,16, \mathrm{p}<0,001)$, raiva $(\mathrm{r}=0,28, \mathrm{p}<0,001)$ e hostilidade $(\mathrm{r}=0,16, \mathrm{p}<0,001)$. O mesmo foi observado para a busca de sensação a novidade e o comportamento agressivo físico $(\mathrm{r}=0,28, \mathrm{p}<0,001)$, raiva $(\mathrm{r}=0,25, \mathrm{p}<0,001)$ e hostilidade $(\mathrm{r}=0,17, \mathrm{p}<0,001)$. Vale destacar, como dado adicional, que a dimensão Busca de Sensação (considerando o somatório de todos os itens da escala) apresentou, também, escores correlacionais positivos com o comportamento agressivo físico $(\mathrm{r}=$ $0,39, \mathrm{p}<0,001)$, verbal $(\mathrm{r}=0,16, \mathrm{p}<0,001)$, raiva $(\mathrm{r}$ $=0,30, \mathrm{p}<0,001)$ e hostilidade $(\mathrm{r}=0,17, \mathrm{p}<0,001)$ (ver Tabela 2). 


\section{Tabela 2 - Correlação entre a busca de sensação e comportamentos agressivos}

\begin{tabular}{lccc}
\hline & \multicolumn{3}{c}{ Busca de Sensação } \\
\cline { 2 - 4 } Comportamento & Intensidade & Novidade & Bs \\
\hline Físico & $0,41^{*}$ & $0,28^{*}$ & $0,39^{*}$ \\
Verbal & $0,16^{*}$ & $0,08^{*}$ & $0,16^{*}$ \\
Raiva & $0,28^{*}$ & $0,25^{*}$ & $0,30^{*}$ \\
Hostilidade & $0,16^{*}$ & $0,17^{*}$ & $0,17^{*}$ \\
\hline
\end{tabular}

Nota: $* \mathrm{p}<0,01 ; \mathrm{BS}=$ Somatório total dos itens Busca de Sensação.

Ao observar esses resultados e a fim de apresentar maior esclarecimento quanto à configuração representacional entre essas relações, pretendeu-se facilitar a percepção relacional entre essas variáveis como um todo, optou-se na elaboração da Figura 1. Nela são contemplados os resultados da Tabela 1 e 2 , onde são destacadas as relações entre a busca de sensação e o comportamento agressivo. Com isso, é possível perceber na representação de um modelo explicativo uma forma organizada de considerar as correlações entre as variáveis; nelas não somente se encontram as dimensões do comportamento agressivo se inter-relacionando positivamente, em destaque no lado esquerdo da figura, como também, suas relações com os traços da personalidade de busca de novidade e intensidade, as quais se correlacionaram entre si (ver Figura 1).

\section{Figura 1 - Representação gráfica das correlações entre comportamento agressivo e busca de sensação}

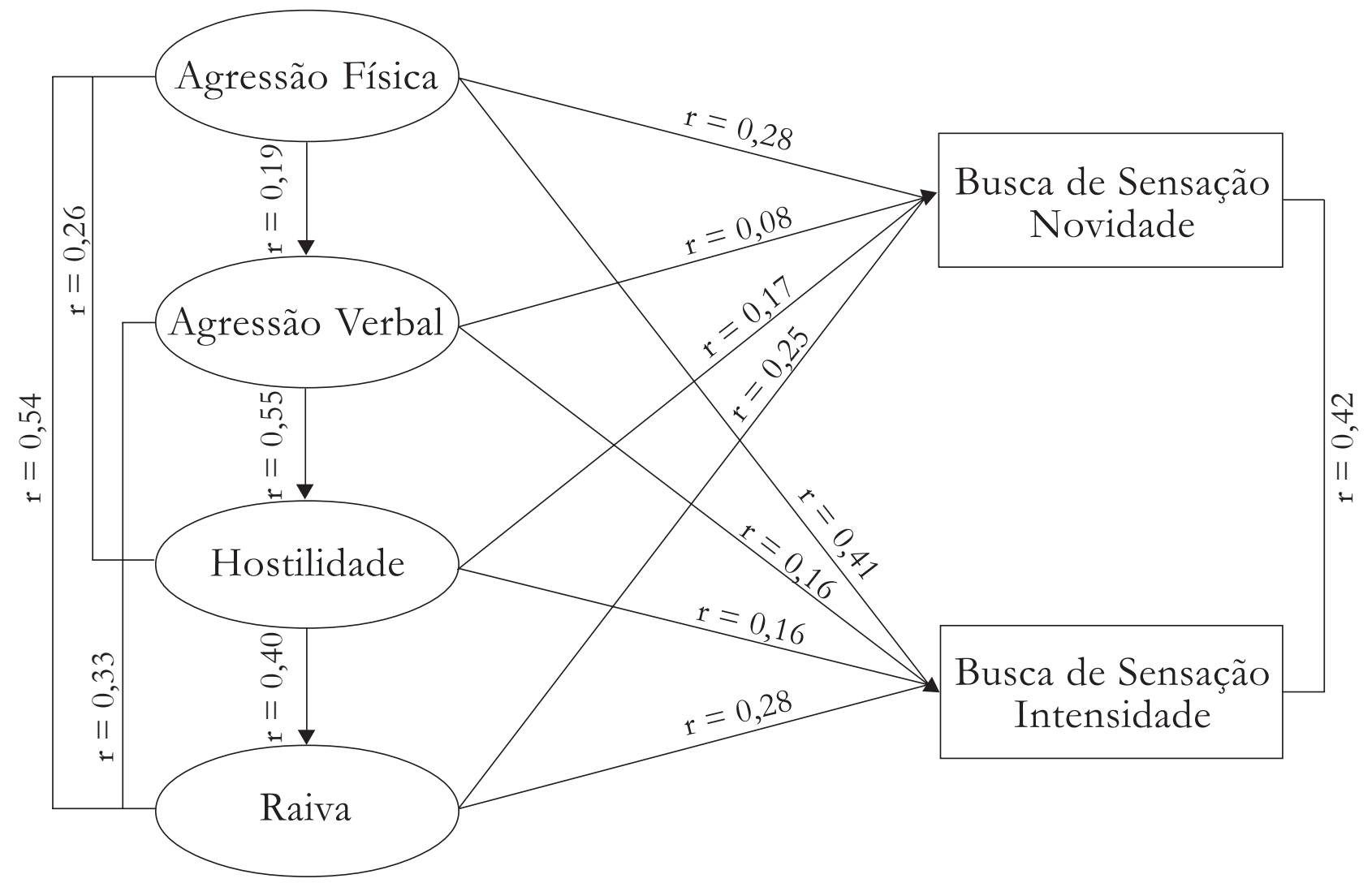

Nota: Todas as correlações foram significativas a um $\mathrm{p}<0,01$. 
Um outro dado adicional deve-se à avaliação da diferença entre as médias de homens e mulheres em relação ao construto busca de sensação e comportamento agressivo; na Tabela 3 é possível visualizá-las: no que diz respeito à busca de intensidade, especificamente, os homens apresentaram uma média superior $(\mathrm{M}=2,58, \mathrm{DP}=0,65)$ à das mulheres $(\mathrm{M}=2,30, \mathrm{DP}=0,67)[\mathrm{t}(403)=4,25, \mathrm{p}<$ $0,01]$; em relação à busca de novidade, os homens também pontuaram mais alto $(\mathrm{M}=2,78, \mathrm{DP}=0,75)$ do que as mulheres $(\mathrm{M}=2,60, \mathrm{DP}=0,64)[\mathrm{t}(404)$ $=2,25, \mathrm{p}<0,01]$. Esses resultados se assemelham aos encontrados por Omar e Uribe (1998), observando que os homens apresentaram médias superiores à das mulheres em ambas as dimensões da busca de sensação. Na mesma Tabela 3, no que diz respeito ao comportamento agressivo físico, os homens apresentaram uma média superior $\left(M_{\text {homem }}\right.$ $=2,53, \mathrm{DP}=0,82)$ à das mulheres $\left(\mathrm{M}_{\text {mulher }}=2,28\right.$, $\mathrm{DP}=0,98)[\mathrm{t}(443)=2,84, \mathrm{p}<0,01]$. Por outro lado, no comportamento agressivo verbal e raiva, as mulheres apresentaram médias superiores à dos homens (respectivamente, para agressão verbal, $\mathrm{M}_{\text {mulher }}$ $=\mathbf{3}, \mathbf{0 1}, \mathrm{DP}=1,05$ e $\mathrm{M}_{\text {homem }}=2,87, \mathrm{DP}=0,98$, $[\mathrm{t}(455)=-2,67, \mathrm{p}<0,05]$; para raiva, $\mathrm{M}_{\text {mulher }}=2,97$, $\mathrm{DP}=1,00$ e $\mathrm{M}_{\text {homem }}=2,61, \mathrm{DP}=0,97,[\mathrm{t}(449)=-$ 2,91, $\mathrm{p}<0,01]$; a dimensão hostilidade não obteve resultado significativo.

\section{Tabela 3 - Diferença entre homens e mulberes em relação a busca de sensação e comportamento agressivo}

\begin{tabular}{|c|c|c|c|c|c|c|}
\hline \multicolumn{7}{|c|}{ Gênero } \\
\hline \multirow[b]{2}{*}{ Busca de Sensação } & \multicolumn{2}{|c|}{ HOMEM } & \multicolumn{2}{|c|}{ MULHER } & \multicolumn{2}{|c|}{ ESTATÍSTICAS } \\
\hline & $\mathbf{M}$ & $\mathrm{DP}$ & $\mathbf{M}$ & DP & gl & $\mathrm{p}<$ \\
\hline Busca de Intensidade & 2,58 & 0,65 & 2,30 & 0,67 & $4,25 \quad 403$ & 0,01 \\
\hline Busca de Novidade & 2,78 & 0,75 & 2,60 & 0,64 & $2,56 \quad 404$ & 0,01 \\
\hline \multicolumn{7}{|l|}{$\begin{array}{l}\text { Comportamento } \\
\text { agressivo }\end{array}$} \\
\hline Físico & 2,53 & 0,80 & 2,28 & 0,98 & $2,84 \quad 443$ & 0,01 \\
\hline Verbal & 2,87 & 0,98 & 3,01 & 1,05 & $-2,67455$ & 0,05 \\
\hline Raiva & 2,61 & 0,97 & 2,97 & 1,00 & $-2,91449$ & 0,01 \\
\hline Hostilidade & 2,99 & 0,90 & 3,03 & 1,02 & $-0,37446$ & n.s. \\
\hline
\end{tabular}

Nos resultados da Tabela 3, é possível refletir no que diz respeito a essas diferenças em termos da administração da conduta, para ambos os gêneros, referindo-se ao desenvolvimento físico, psíquico e social, incluso desde uma perspectiva concreta (orgânica) à abstrata (sociocognitiva). A partir das observações cotidianas, percebe-se que pensamento e ação de homens e mulheres aderem-se à expectativa atribuicional da conduta, mas, também, ao comportamento socialmente desejável; acredita-se que é mais comum, quando se trata de uma conduta instrumental, como é o caso da agressão física, estereotipicamente, ser característica e permissível aos homens (Formiga, 2006; Techio, 1999). Por outro lado, o inverso - as mulheres não serem instrumentalmente agressivas - salvo em ocasiões de violência sexual e outras variações desta, elas revelarem um comportamento agressivo mais expressivo, por exemplo, agressão verbal ou raiva. 
De forma geral, esse julgamento diferenciando o comportamento agressivo para homens e mulheres permeia a manutenção de estereótipos estabelecidos nas relações interpessoais, por meio dos quais são capazes de orientar a conduta de cada gênero, principalmente entre os jovens, por exemplo, justificando-os a partir de traços personalísticos capazes de garantir, e até de impor, em determinadas situações, o controle dessas experiências, considerando sociocognitivamente, o que é mais adequado para homem e para mulher, o que nos leva a refletir em direção do estranhamento das pessoas quando uma mulher reage "agressivamente". Esse fato é possível ao observar as diferenças entre as médias de homens e mulheres em relação à busca de sensações, tanto na busca de novidade quanto intensidade, os homens pontuaram mais alto do que as mulheres. Parece ser que eles tendem a buscar com maior freqüência e potência dessas experiências em correr risco. Esse fato, de acordo com a teoria de Arnett (1994) e Omar e Uribe (1998), não se trata apenas de uma predisposição ao risco, mas também que a saliência nestas dimensões da busca de sensação se deve ao processo de socialização parental e de pares de iguais.

Para tanto, não precisa ir muito longe para se observar as práticas e o discurso apresentados pelos pais e colegas juvenis, no investimento de uma atividade desafiadora que envolva ambos os gêneros, estas são na maioria das vezes carregadas de uma estereotipia feminilizada quanto ao homem que não enfrenta ou submete a competição proposta pelos adolescentes homens provocando moral e socialmente os outros; por outro lado, as mulheres são também floreadas de adjetivos de inferioridades, tornando-as simbolicamente limitadas e incapazes para realizar a competição ou risco proposto pelos homens, justificados por estes últimos como uma condição natural desinibidora que eles têm vinculado a dimensão psicotista defendida por $\mathrm{H}$. J. Eysenck (Omar, 2001; Omar, \& Uribe, 1998).

No que diz respeito aos resultados encontrados entre as variáveis da busca de sensação e comportamento agressivo, este foi corroborado; não apenas em termos do objetivo proposto neste estudo, mas também em comparação com outros que seguiram semelhante raciocínio. Obviamente, os estudos anteriores se centravam em perspectivas teóricas diferentes quanto ao construto da busca de sensação, a maioria utilizava a concepção e o instrumento de M. Zuckerman, porém, convergindo para um núcleo teórico central: os adolescentes que buscam correr risco incluem-se numa característica personalística que aponta para taxonomias de instabilidade emocional, afetos negativos, irritabilidade etc., relacionando-se com o comportamento agressivo físico, a hostilidade e a raiva (Arnett, 1994; Omar, \& Uribe, 1998; Zuckerman, 1971).

Com base na teoria de Arnett, os achados no presente estudo vem enfatizar que, quanto maior a busca de intensidade e novidade, consecutivamente, maior probabilidade de que os jovens possam apresentar um comportamento agressivo físico, verbal, raiva ou hostilidade. Ao afirmar esse fato, não somente está se referindo à característica intrínseca do sujeito relacionado aos traços de personalidade, mas aos processos de socialização, os quais podem incentivar a experiência do risco, por meio da busca intensa e nova das sensações (Joiremann, Anderson, \& Strathman, 2003). O que a perspectiva da busca de sensação vem apontar na literatura em geral, e até no senso comum, é que o jovem tem uma necessidade latente em expandir seu mundo ideal e "real" por meio do comportamento questionador e instrumental, carregado de emoções com características ansiosas, estando disposto a convites pessoais ou sociais a fim de viver as novas descobertas, ensejando sentilas "a flor da pele", visceralmente os sentidos de excitação, porém, não se conscientizando dos possíveis perigos que essas situações possam trazer para eles e para as pessoas com quem convivem.

Esta condição de conscientização se daria a partir de uma análise das variáveis de intensidade do afeto durante o desenvolvimento da puberdade tendo um monitoramento e apoio familiar quanto à conduta e controle das proximidades dos fatores de risco para os adolescentes, desde um comportamento impulsivo ao extrovertido, conceituado na maioria das vezes como da fase da adolescência ou "coisa de jovem" (Martin et al., 2002; Omar, 2001). É bem possível que em tal discurso passe despercebido - justamente porque quando socializado o jovem se desindividualiza, isto é, perde a identidade pessoal para a intergrupal possibilitando sua inserção nas condutas tangenciadoras das normas sociais, etc. (Urra, 2003). De outra maneira, quando os pares socionormativos, por exemplo, pai, mãe e professor, ao participarem e atentarem para as mudanças de humor e comportamento, pelo menos aquelas que tangenciam, excessivamente, as influências 
hormonais e sociais, não somente teriam o poder de inibir os fatores potencializadores das variáveis de risco juvenil, bem como a orientação familiar em relação a uma melhor medida na busca de experimentar novidade ou intensificar sensações durante esse explosivo período de desenvolvimento (Formiga, 2005).

\section{Conclusões}

Resumidamente, é possível refletir, ao enfatizar o estudo de Martin et al. (2002) e de Formiga, Yepes e Alves (2005), quando no cotidiano considera-se o discurso do jovem flagrado em atitudes permeadoras da delinqüência (por exemplo, agressão, uso de droga, condutas delitivas, etc.) é comum o fator curiosidade, influência dos outros colegas, provocação pessoal ou relacionado ao grupo de iguais, justificando como uma descoberta das experiências sensíveis paralelas à necessidade em assumir características da adolescência ou aquela falsa modéstia de ser adulto "independente", ou até, por desentendimentos e falta de atenção socioafetiva familiar.

Ao salientar esses estudos, pretende-se destacar esse fenômeno como multivariado, em cadeia em vez de linear e isolado, tanto na intenção de predizer as condutas sociais a partir das variáveis personalísticas, quanto esta última originar-se na dinâmica da socialização, como propõem Arnett (1994; 1996; Omar, \& Uribe, 1998). Por fim, apesar de ter corroborado as hipóteses e atendido aos objetivos do presente estudo, este não pode responder definitivamente o problema, mas sim contribuir como mais uma peça no quebra-cabeça do fenômeno da delinqüência.

Sendo assim, é preciso reconhecer limites no estudo, por exemplo, dentre eles: 1 - seria de muita utilidade um estudo comparativo com diferentes grupos permeadores das condutas desviantes, isto é, aqueles que sofrem penalização e estão em instituições de privação de liberdade e os que não estão sob pena da lei e não apresentam história de delinqüência a fim de avaliar a variabilidade da busca de sensação, bem como, os pares e contexto socializador que influenciou os comportamentos de risco; 2 - avaliar os jovens em diversas classes econômicas e sua variação na busca de sensação, incluindo outras variáveis, por exemplo, hábitos de lazer e prática parental; 3 - poderia ser também importante o delineamento de um estudo multidisciplinar, no qual fosse possível contemplar um estudo clínico, experimental e correlacional, capaz de configurar uma potencialidade do comportamento agressivo entre os jovens de diversas representações socioeconômicas.

\section{Referências}

Allport, G. W. (1973). Personalidade: Padrões e desenvolvimento. São Paulo: EPU.

Anastasi, A., \& Rubina, S. (2000). Testagem psicológica. Porto Alegre: Artmed.

Anderson, C. A., \& Bushman, B. J. (2002) Human aggression. Annual Reviews Psychology, 53, 27-51.

Andreu, J. M. R.; Peña, M. E. F.; Graña, J. L. G. (2002). Adaptación psicométrica de la versión española del cuestionario de agresión. Psicothema, 14(2), 476-482.

Arnett, J. (1994). Sensation seeking: a new conceptualization and a new scale. Personality and Individual Differences, 16(2), 289-296.

Arnett, J. J. (1996). Sensation seeking, aggressiveness and adolescent reckless behavior. Personality and Individual Differences, 20(6), 663-702.

Aronson, E., Wilson, T. D., \& Arket, R. M. (2002). Agressão: Por que agredimos outras pessoas? In Elliot Aronson, Timothy D. Wilson e Robin M. Akert (Org.), Psicologia Social (pp.270-281). Rio de Janeiro: LTC.

Ávila, A. E., Rodríguez, S. C., \& Herrero, J. R. S. (1997). Evaluación de la personalidad patológica: Nuevas perspectivas. In E. Cordero (Org.), La evaluación psicológica en el año 2000. Madrid: TEA.

Barbaranelli, C., \& Caprara, G. V. (1996). How many dimensions to describe personality? A comporison of Cattel, Comrey, and the Big Five taxonomies of personality traits. European Review of Applied Psychology, 46(1), 15-24.

Benet-Martínez, V., \& John, O. P. (1998). Los Cinco Grandes across cultures and ethnic groups: multitrait multimethod analyses of the Big Five in Spanish and English. Journal of Personality and Social Psychology, 75, 729-750. 
Bryant, F. B., \& Smith, B. D. (2001). Refining the architecture of aggression: A measurement model for the Buss-Perry aggression questionnaire. Journal of Research in Personality, 35, 138-167.

Buss, A. H., \& Perry, M. (1999). The aggression questionnaire. Journal of Personality and Social Psychology, 63(3), 452-459.

Caprara, G. V., Barbaranelli, C., \& Zimbardo, P. G. (2003). Understanding the complexity of human agression: Affective, cognitive, and social dimensions of individual differences in propensity toward aggression. European Journal of Personality, 10, 133-155.

Cloninger, S. C. (1999). Teorias da personalidade. São Paulo: Martins Fontes.

Coelho Junior, L. L. (2001). Uso potencial de drogas em estudantes do ensino médio: Suas correlações com as prioridades axiológicas. Dissertação (Mestrado em Psicologia Social). Universidade Federal da Paraíba, João Pessoa.

Collani, G., \& Werner, R. (2005). Self-related and motivational constructs as determinants of aggression: An analysis and validation of a German version of the Buss-Perry aggression questionnaire. Personality and Individual Differences, 38, 1631-1643.

Cordero, E. (1997). La evaluación psicológica en el año 2000. Madrid: TEA.

Costa, P. T., \& McCrae, R. R. (1992). Four ways five factors are basic. Personality and Individual Differences, 13, 653-665.

Díaz, L. R. (1998). Cultura y Personalidad: Rasgo universales e idiosincrásicos. In Alcaraz y Bouzas (Coord.). Las aportaciones mexicanas a la psicología. México: UNAM.

Engler, B. (1984). Personality theories: An Introduction. Boston, MA: Houghton Mifflin.

Eysenck, H. J. (1981). Impulsiveness and anti-social behavior in children. Current Psychological Research, 1, 31-37.

Formiga, N. S. (2006). Gênero e variações emocionais básicas: Diferenças entre homens e mulheres nos antecedentes das emoções de cólera, alegria e tristeza. Revista Científica Eletrônica de Psicologia, 3, (6), 1-16.
Formiga, N. S. (2005). Os componentes variantes da violência: Convergência entre comportamento agressivo e conduta anti-social e delitiva em jovens. In Semana do Psicólogo, 6. João Pessoa-PB.

Formiga, N. S., \& Gouveia, V. V. (2003). Adaptação e validação da escala de condutas anti-sociais e delitivas ao contexto brasileiro. Revista Psico, 34(2), 367-388.

Formiga, N. S., \& Gouveia, V. V. (2005). A Predição das Condutas Anti-sociais e Delitivas em Jovens Baseado nos Valores Humanos. Revista de Psicologia UNC, 2(2), 103-114.

Formiga, N. S., Saraiva, C. P., Pequeno, N. L. P., Trigueiro, E. S. O., Joca, E. C., Leal, R. S., et al. (2005). Diferenças individuais e comportamentos desviantes:Correlatos dos cinco grandes fatores da personalidade e conduta anti-social e delitiva. In Anais, 1. Congresso Latino-Americano de Psicologia em São Paulo, 1. São Paulo-SP.

Formiga, N. S., Teixeira, J., Curado, F., Lüdke, L., \& Oliveira, A. R. N. (2003). A predisposição das condutas anti-sociais e delitivas a partir dos trações de personalidade. In: 33. Reunião Anual da Sociedade Brasileira de Psicologia. Belo Horizonte.

Formiga, N. S., Trigueiro, E. S. O., Melo, C. F., \& Dourado, J. L. G. (2006). A busca de sensação e as variações condutuais da violência juvenil: A experiência em correr. risco como explicação das condutas desviantes, comportamento agressivo e uso potencial de drogas. In 58 Reunião Anual da SBPC: Semeando Interdisciplinaridade. Florianópolis.

Formiga, N. S., Yepes, C., \& Alves, I. (2005). Correlatos entre traços de personalidade e afiliação com pares sociais: Reflexões a respeito da formação personalística em jovens. In Anais, 4 Congresso Científico do Ceulp-Ulbra: Ética e Ciência. Palmas-TO.

Fossati, A., Maffeti, C., Acquarini, E., \& Di Ceglie, A. (2003). Multigroup confirmatory component and factor analyses of the Italian version of the aggression questionnaire. European Journal of Psychological Assessment, 19(1), 54-65.

Frías, M. A., López-Escobar, A, E., \& Díaz-Méndez, S. G. (2003). Predictores de la conducta antisocial juvenil: Un modelo ecológico. Estudos de Psicologia, 8(1), 15-24. 
Gazzaniga, M. S., \& Heatherton, T. F. (2005). Personalidade. In: Michael S. Gazzaniga e Todd F. Heatherton (Org.), Ciência Psicológica: Mente, cérebro e comportamento. (pp. 486496). Porto Alegre: Artmed.

Heaven, P. C. L., Newbury, K., \& Wilson, V. (2004). The Eysenck psychoticism dimension and delinquent behaviours among non-criminals: Changes across the lifespan? Personality and Individual Differences, 36, 1817-1825.

Hutz, C. S., Nunes, C. H., Silveira, A. D., Serra, J., Anton, M., \& Wieczorek, L. S. (1998). O desenvolvimento de marcadores para a avaliação da personalidade no modelo dos cinco grandes fatores. Psicologia: Reflexão e Crítica, 11(2), 395-411.

Joireman, J., Anderson, J., \& Strathman, A. (2003). The aggression paradox: Understanding links among aggression, sensation seeking and the consideration of future consequences. Journal of Personality and Social Psychology, 84(6), 1287-1302.

Kristensen, C. H., Lima, J. S., Ferlin, M., Flores, R. Z., \& Hackmann, P. H. (2003). Fatores etiológicos da agressão física. Estudos de Psicologia, 8(1), 175-184.

Martin, C. A., Thomas, H. K., Rayens, M. K., Brogli, B. R., Brenzel, A., Smith, W. J. et al. (2002). Sensation seeking, puberty and nicotine, alcohol and marijuana use in adolescence. Journal Academic Child Adolescence Psychiatry, 41(12), 1495-1502.

McAdams, D. P. (1992). The Five-factor personality profiles. Journal of Personality Assesssment, 60, 329-361.

Meesters, C., Muris, P., Bosma, H., Schouten, E., \& Beuving, S. (1996). Psychometric evaluation of the Dutch version of the aggression questionnaire. Behavior Research and Therapy, 34, 839-843.

Michel, G., Mouren-Siméoni, M-C., Perez-Diaz, F., Falissard, B., Carton, S., \& Jouvent, R. (1999). Construction and validation of a sensation seeking scale for adolescents. Personality and Individual Differences, 26, 159-174.

Michener, H. A., Delamater, J. D, \& Myers, D. J. (2005). Agressão. In: H. Andrew Michener, John D. DeLamater e Daniel J. Myers (Org.), Psicologia Social. (pp. 329-357). São Paulo: Thomson.
Mussen, P. H., Conger, J. J., Kagan, J. E, \& Huston, A. C. (1995). Desenvolvimento e personalidade da criança. (3a ed.). São Paulo: Harbra.

Omar, A. G. (2001). Búsqueda de sensaciones e intensidad de afecto. In M., Hernández González. (Org.), Motivación animal y humana. México: El Manual Moderno.

Omar, A., \& Uribe, H. D. (1998). Dimensiones de personalidad y busqueda de sensaciones. Psicologia: Teoria, Investigação e Prática, 3, 257-268.

Paunonen, S. V. (1998). Hierarchical organization of personality and prediction of behavior. Journal of Personality and Social Psychology, 74(2), 538-556.

Peabody, D. (1987). Personality dimensions through trait inferences. Journal of Personality and Social Psychology, 46(2), 384-403.

Pervin, L. A., \& John, O. P. (2004). Personalidade: teoria e pesquisa . Porto Alegre: Artmed.

Queiroga, F., Formiga, N. S., Jesus, G. R., Gouveia, V. V., \& Andrade, J. M. (2001). Desejabilidade social e Personalidade. In Anais, 31. Reunião Anual de Psicologia: A Construção da Psicologia na Pesquisa e no Ensino, Rio de Janeiro-RJ.

Robinson, J. P, Shaver, P. R., \& Wrightsman, L. S. (1991). Measures of personality and social psychological attitudes. San Diego: Academic Press.

Romero, E., Luengo, M. A., \& Sobral, J. (2001). Personality and antisocial behavior: Study of temperamental dimensions. Personality and Individual Differences, 31, 329-348.

Saudino, K. J., \& Plomin, R. (1996). Personality and Behavior Genetics: Where Have Been and Where Are We Goin? Journal of Research in Personality, 30, 335-347.

Sharpe, J. P., \& Desai, S. (2001). The revised NEO personality inventory and the MMPI-2 psycholopatology five in the prediction of aggression. Personality and Individual Differences, 31, 505-518.

Sobral, J. (1996). Psicología social jurídica. In: J. L. Álvaro, A. Garrido, \& J. R. Torregrossa (Org.), Psicología Social Aplicada. Madrid: McGraw-Hill. 
Stephenson, G. F. (1990). Psicología Social Aplicada. In: M. Hewstone, W. Stroebe, J. P. Codol, \& G. M. Stephenson (Org.), Introducción a la psicología social: Una perspectiva europea. Barcelona: Ariel.

Techio, M. E. (1999). Conhecimento Social das Emoções: Um estudo em termos de fatores cognitivos e culturais. Dissertação de Mestrado em psicologia social, Universidade Federal da Paraíba, Paraiba.

Tranah, T., Harnett, P. E, \& Yule, W. (1998). Conduct disorder and personality. Personality and Individual Differences, 24, 741-745.

Tremblay, P. F., \& Ewart, L. A. (2005). The Buss and Perry aggression questionnaire and its relations to values, the big five, provoking hypothetical situations, alcohol consumption patterns, and alcohol expectancies. Personality and Individual Differences, 38, 337-346.

Trzop, B. M. (2000). The Big Five: Taxonomy of trait theory. (On line). Recuperado em 24 dez. 2005: http//www.personality research.org/ papers/popkins.html.

Urra, J. (2003). Adolescencia y violencia: Topicos y realidades. Revista de Estudios de Juventud, 62(3), 11-20.
Williams, T. Y., Boyd, J. C., Cascardi, M. A., \& Poythress, N. (1996). Factor structure and convergent validity of the aggression questionnaire in an offender population. Psychological Assessment, 8(4), 398-403.

Zhang, L., Welt, J. W., \& Wieczorek, W. F. (1999). Young, drug use, and delinquency. Journal of Criminal Justice, 27, 101-109.

Zuckerman, M. (1971). Dimensions sensation of seeking. Journal of Consulting and Clinical Psychology, 36, 45-52.

Zuckerman, M., Eysenck, S. B. G., \& Eysenck, H. J. (1978). Sensation seeking in England and America: Cross-cultural, age and sex comparisons. Journal of Consulting and Clinical Psychology, 46, 139-149.

Recebido em: 20/03/2007

Received in: 03/20/2007

Aprovado em: 02/04/2007

Approved in: 04/02/2007 